\title{
Surgical considerations in patients with lumbar spinal root anomalies
}

\author{
M N Pamir MD, ${ }^{1}$ M M Özek MD, ${ }^{2}$ A F Özer MD, G E Keleş MD, C Erzen MD \\ ${ }^{1}$ Professor of Neurosurgery and Director, ${ }^{2}$ Assistant Professor of Neurosurgery, \\ ${ }^{3}$ Associate Professor of Neurosurgery, ${ }^{4}$ Resident in Neurosurgery, Department of \\ Neurosurgery, 5 Professor of Radiology and Director, Department of Radiology, Marmara \\ University Hospital, Istanbul, Turkey.
}

Lumbosacral nerve root anomalies are rare and can cause diagnostic confusion. In this report we present 12 patients with lumbar root anomalies. Emphasis is placed on preoperative neuroradiological evaluation and the surgical implications of these anomalies.

Key words: lumbosacral nerve roots; root anomalies; myelography; computerized tomography; operative surgery.

\section{Introduction}

Lumbosacral nerve root anomalies, which were first reported by Zagnoni, ${ }^{1}$ are a rare group of anatomical anomalies. There are only a few reports on the myelographic and computerized tomographic appearance of such cases. ${ }^{2-6}$

According to White et al, ${ }^{6}$ root anomalies are present in only $1.3 \%$ of all patients operated on with a diagnosis of lumbar disc herniation. In these rare cases the results obtained using classical surgical techniques are poor, and the incidence of root injuries is high. ${ }^{7}$ The most important factor is preoperative diagnosis of the anatomical variation by either myelography or computerised tomography $(\mathrm{CT})$.

The aim of this report is to present 12 cases of lumbar root anomaly and to discuss our findings in comparison to cases described in the literature.

\section{Material and method}

Two of the patients were male (17\%) and 10 were female $(83 \%)$. Their ages ranged between 17 and 53 with a mean of 38 years.

Three $(25 \%)$ complained of sciatica whilst the remaining 9 patients $(75 \%)$ had low back pain in addition to sciatica.

Neurological examination revealed deficits due to L5 root compression in 9 cases ( $75 \%)$ and $\mathrm{S} 1$ root compression in 3 cases
(25\%). A negative Laseque's sign was present in 10 cases $(83 \%)$.

All of our patients were studied by CT scan, and lumbar root anomalies were determined in $9(75 \%)$ of them. In $3(25 \%)$, the root could not be detected at the level of the disc on one side. Five patients $(42 \%)$ had a soft tissue lesion with the same density value as the thecal sac, obliterating the epidural fat. The CT appearance of 4 cases $(33 \%)$ was similar to that of an extruded disc fragment.

Myelography was performed on all of the patients using a water soluble contrast medium (Omnipaque, Iohexol $300 \mathrm{mgI} / \mathrm{ml}$, Nycomed AS, Oslo) and root anomalies were demonstrated in each patient. Two patients with diagnostic problems were re-studied by CT following intrathecal contrast material injection.

Laminectomy, facetectomy and pediculectomy were performed on all patients operated on with the clinical diagnosis of root anomaly. Nine cases $(75 \%)$ having an associated herniated nucleus pulposus without free fragments underwent discectomy. In the remaining 3 cases (25\%) severe stenosis of the lateral recess was disclosed. There was no mortality or operative complication. Postoperative outcome of all of the patients was uneventful. Follow up neurological examinations up to 2 years were in the normal range.

After neuroradiological examination and 
surgical findings, nerve root anomalies were grouped according to the classification proposed by Neidre and Macnab ${ }^{5}$ (Table I). Five of our patients $(42 \%)$ were found to be Type I; 4 (33\%) Type II; a single case $(8 \%)$ Type III; and 2 cases $(17 \%)$ were classified as combined type (Figs 1-5).

Table I Distribution of our cases according to Neidre and Macnab's classification

\begin{tabular}{ll}
\hline Type & Number of patients \\
\hline Ia & 4 \\
Ib & 1 \\
IIa & 2 \\
IIb & 2 \\
III & 1 \\
Combined & 2 \\
Total & 12 \\
\hline
\end{tabular}

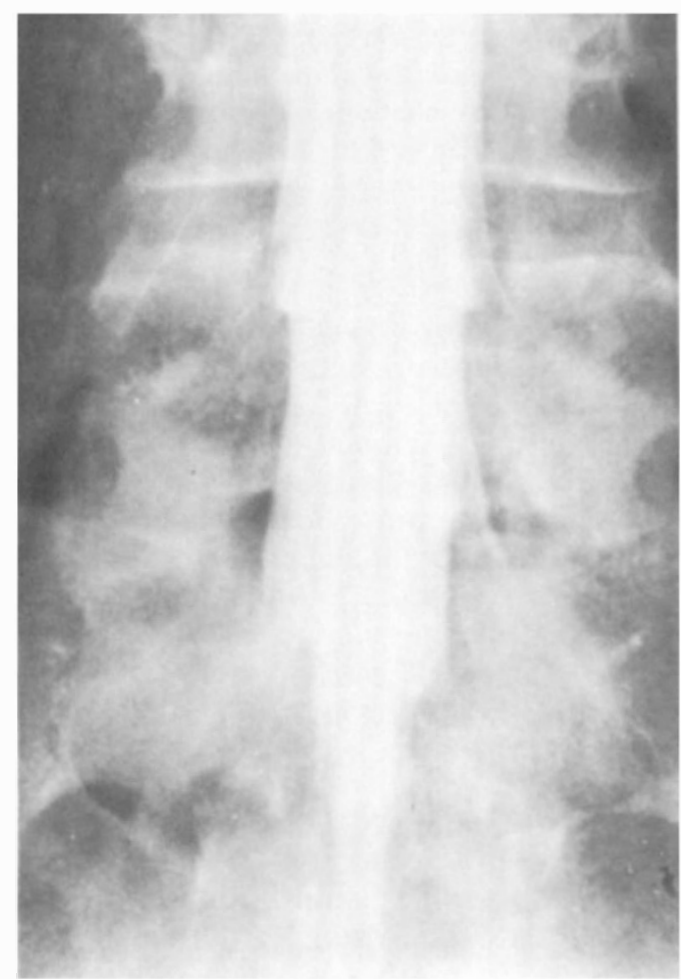

Figure 1a AP myelogram of a Type Ia case. S1 and S2 arise from a common dural sheath.

\section{Discussion}

Lumbar nerve root anomalies were first classified as conjoined roots, transverse

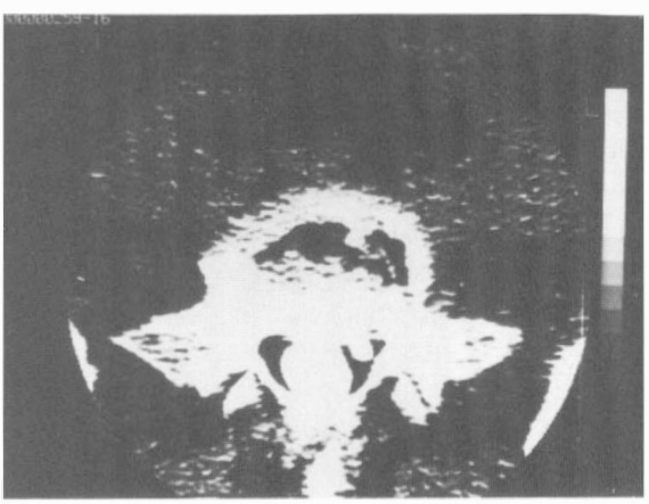

Figure 1b Absence of the right S1 root was noticed at the L5-S1 level on myelo-CT. S1 and S2 roots leave the dural sac at a lower level.

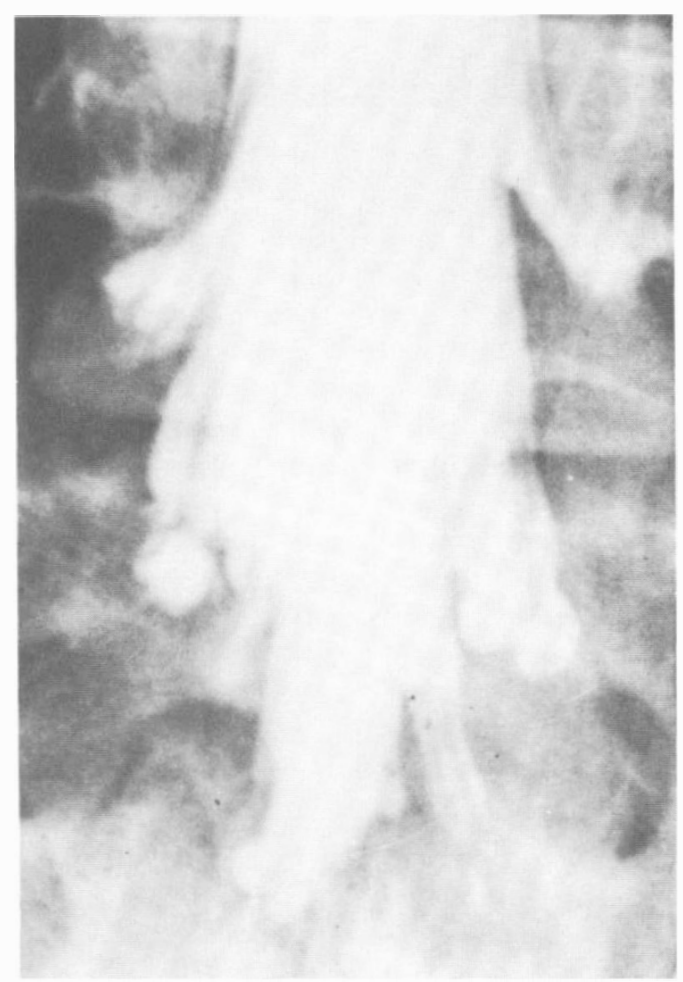

Figure 2a AP myelogram of a Type Ib case. Two nerve roots arise from a common dural sheath where one of the roots exits at right angle to the dural sheath on the right side. 


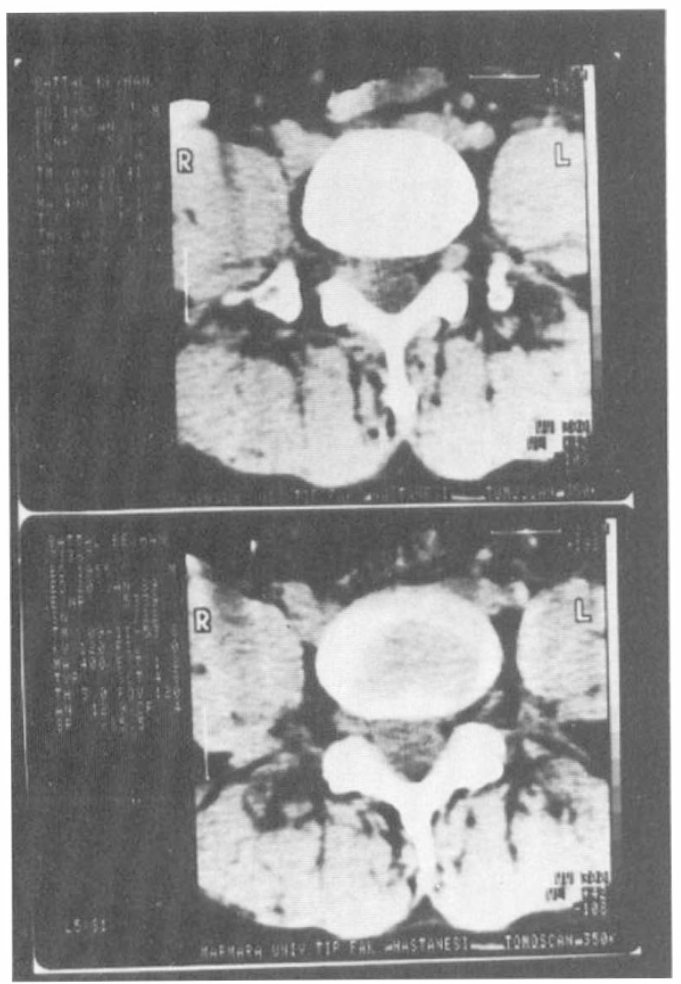

Figure 2b CT scan at the L5-S1 level revealed increased soft tissue density on the right side. Epidural fat tissue was detectable only on the left side.

roots and anastomotic roots. ${ }^{7}$ In 1983 Neidre and Macnab ${ }^{5}$ proposed a more useful classification with 4 groups. Using the latter classification, most of our cases $(75 \%)$ were
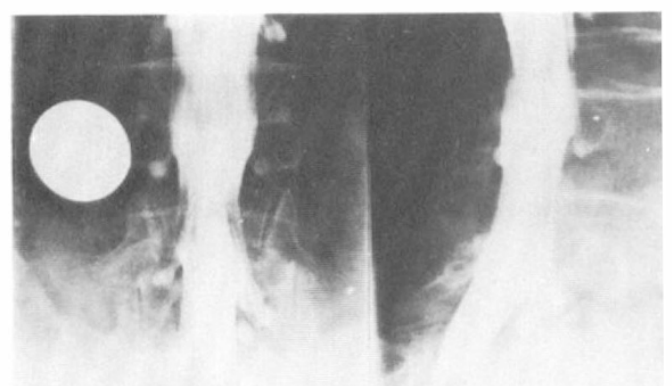

Figure 3a AP and lateral myelograms of a Type IIa case where 2 nerve roots exit through one foramen on the left side without any evidence of disc herniation.

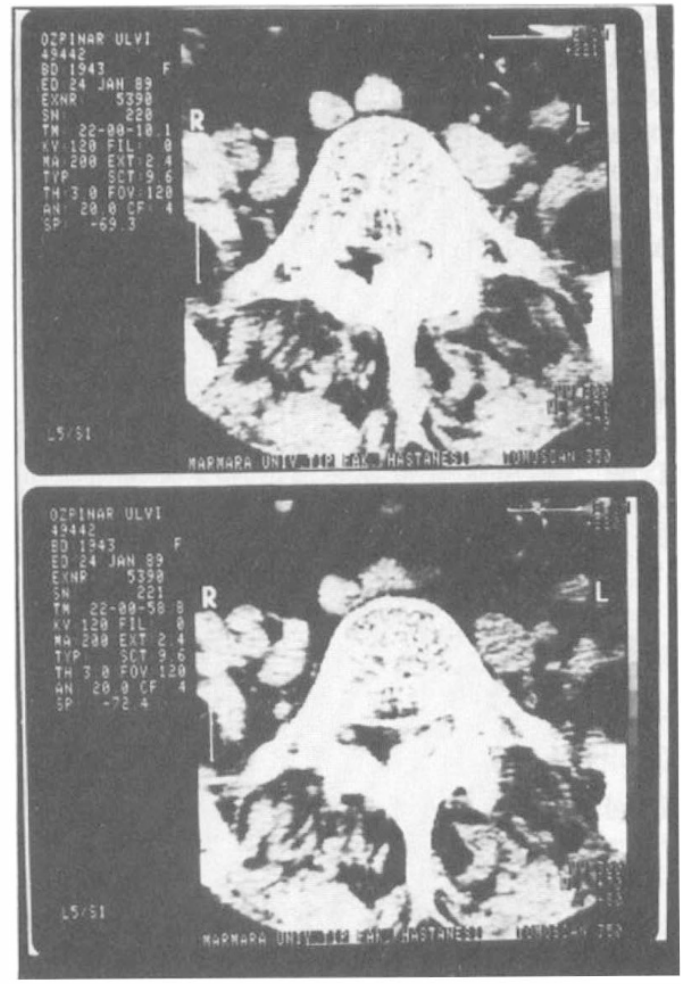

Figure 3b Myelo-CT of the same case at the L5-S1 level revealed obvious epidural fat tissue on the right side and accumulation of the contrast material on the left side without evidence of any fat tissue.

found to be Type I and Type II, being in accordance with previous reports. ${ }^{5}$

From the clinical viewpoint, although Cannon et $a l^{7}$ claimed that symptoms appear as a result of tension on the abnormal root, our experience and that of others ${ }^{2}$ suggest that root anomalies do not cause low back pain or sciatica unless there is an associated disc herniation or lateral recess stenosis. In our 12 cases of root anomaly an additional disc herniation was present in 9 and severe stenosis of the lateral recess in the remaining 3 patients.

Preoperative diagnosis of root anomalies is very important because standard surgical intervention will be insufficient to provide adequate decompression. ${ }^{3}$ Additionally, being unaware of the presence of such an anomaly may lead to extensive neural traction during exploration, and anomalous 


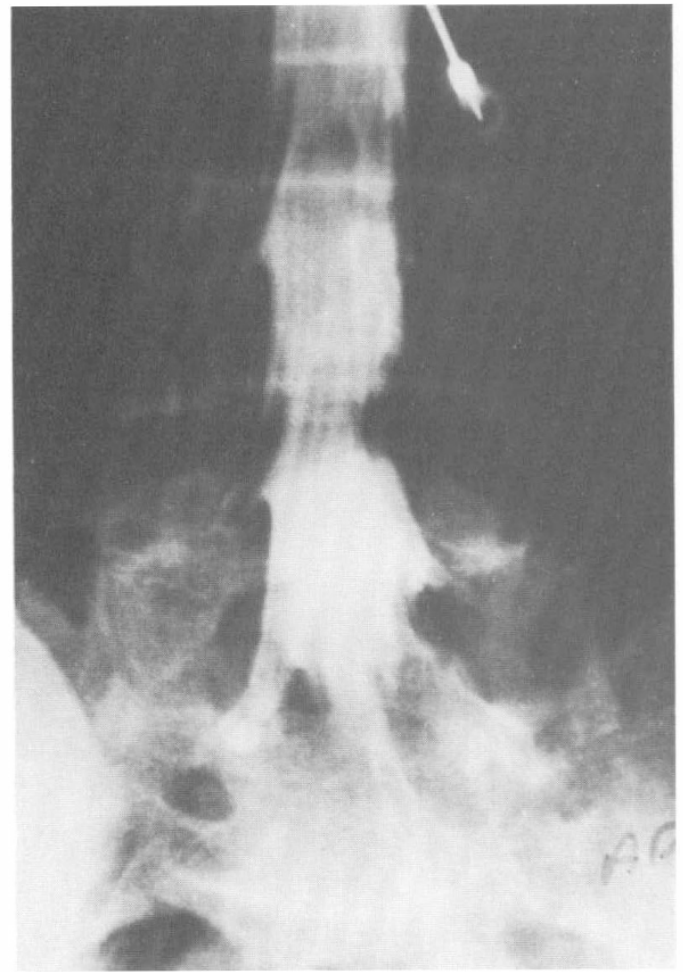

Figure 4a AP myelogram of a Type IIa case with lateral disc herniation on the left side at L4-5 level. Left L5 and S1 roots exit through one foramen and one root canal was unoccupied.

roots may be traumatized. This was first pointed out by Cannon et $a l^{7}$ who reported unsatisfactory results with hemilaminotomy and discectomy in patients with lumbar disc herniation and conjoined roots. Similarly White $e a^{6}{ }^{6}$ stated that only a $30 \%$ success rate may be achieved with a standard surgical technique and emphasised the importance of additional pediculectomy. We share this view and believe that hemilaminotomy and discectomy do not provide sufficient decompression in such cases. For this reason we performed laminectomy and facetectomy with excision of the pedicles and all compressing tissues.

During the last 3 years, reexploration was required in 10 patients $(2.5 \%)$, who had previously been operated on for lumbar disc herniation in our clinic. Root anomalies were determined in $2(20 \%)$ of them.

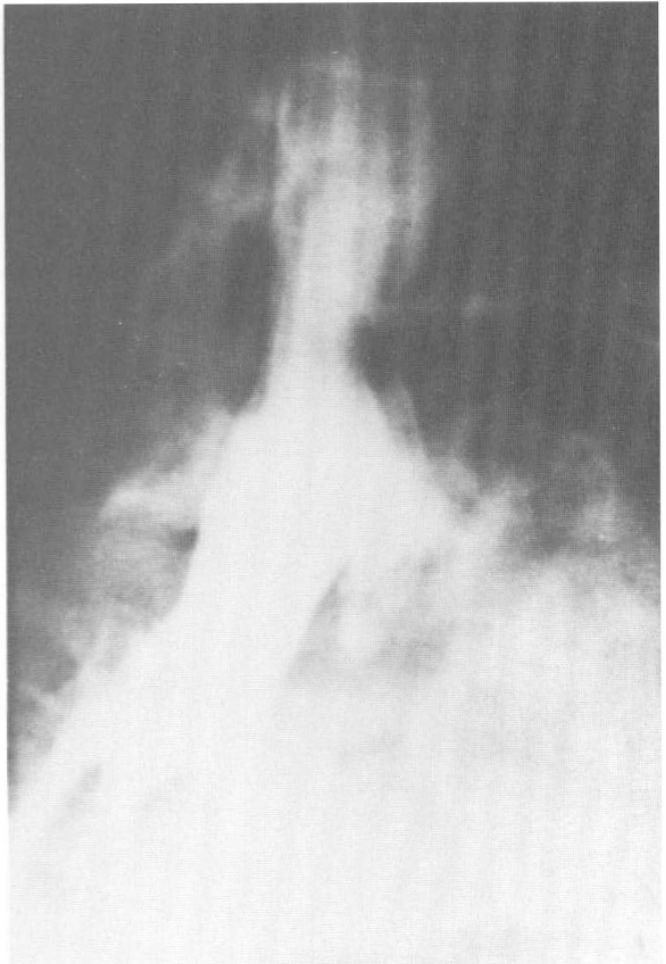

Figure 4b In the lateral lumber myelogram, the lateral disc herniation and the parallel course of both nerve roots were detected.

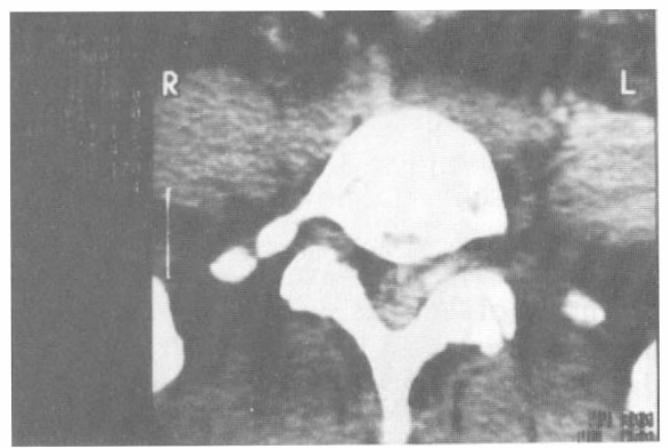

Figure 4c CT scan at the L5-S1 level revealed soft tissue density of equal density with the techal sac.

Following wide decompression, both these patients were relieved of symptoms. For this reason, we suggest that root anomalies should be kept in mind in patients who have had an unsatisfactory lumbar disc herniation operation, and they must be reevaluated 


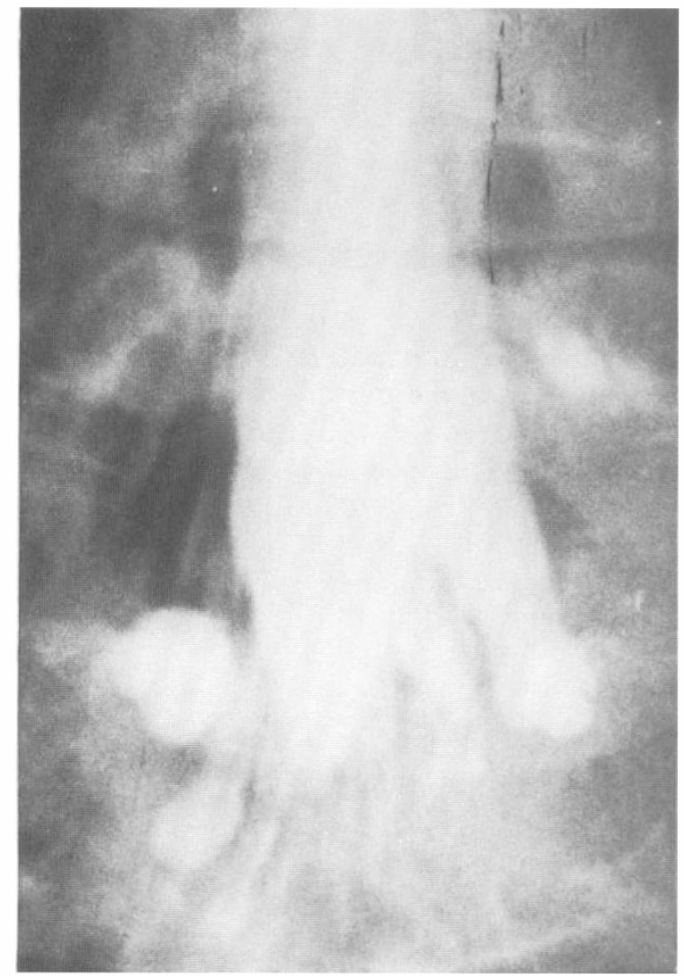

Figure 5a In the myelogram of a Type IIb case we can detect nerve roots in all foramina but one foramen contains 2 separate roots on the left side.

retrospectively for this rare entity. These 12 cases with root anomalies comprise only $2.5 \%$ of the 400 cases who underwent lumbar disc herniation operations during the same period.

Spinal CT is now routinely used in the diagnosis of lumbar disc herniation and is highly sensitive and specific. ${ }^{3,4,8}$ Except for the 2 cases whose root anomaly was not initially detected, we were able to diagnose root anomalies in 10 cases with a CT scan preoperatively.

It should always be remembered that root anomalies may cause false positive results in cases with lateral disc protrusion or neuroforaminal extrusion. ${ }^{4}$ In these patients an obvious increase in soft tissue density is present at the pathological site. Additionally, obliteration of the ipsilateral neuroforaminal fat tissue and lateral recess is detected. In 5 of our cases $(42 \%)$, frank
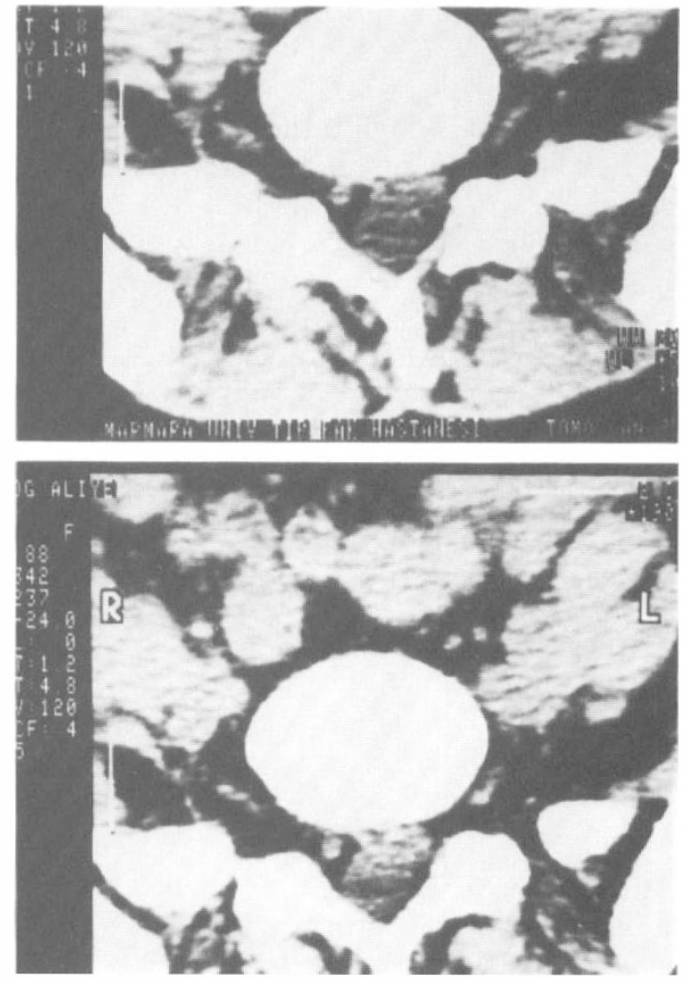

Figure 5b CT scan of the same case revealed obvious soft tissue density on the left side where we can detect the right S1 root within the epidural fat tissue.

diminution of the fat tissue at the anomalous side, indicating a root anomaly, was determined.

Density measurement should be performed in all suspicious cases. ${ }^{4.8}$ If the soft tissue in the lateral recess of neuroforamen has a higher density than $40-50 \mathrm{HU}$, it will most likely represent lumbar disc herniation. ${ }^{4}$ But a density lower than $40 \mathrm{HU}$ may indicate the presence of root anomaly. In 2 of our cases we made the diagnosis with the aid of density measurements. If the density is similar to a free disc fragment, and exploration of the disc space is negative, the possibility of a root anomaly should be considered. Besides, spinal roots leaving the thecal sac must be examined carefully on both sides at each level and lack of a root on one side should implicate root anomaly. Helms et $a l^{4}$ emphasised this point and claimed that differentiation between a con- 
joined nerve root and a herniated nucleus pulposus may be made by changing the scanning density in CT scans. Unilateral lack of a root led us to the diagnosis in 3 of our patients. With these cases our rate of diagnosis in root anomalies by $\mathrm{CT}$ has reached $83 \%$.

Myelographic characteristics of root anomalies are well described. ${ }^{2,8,9}$ Myelography should be performed on every patient where a CT scan suggests a root anomaly. ${ }^{3}$ Besides verifying anatomical variations present on the CT scan, myelography is also efficient in determining the type of anomaly and disclosing variations which could not be noticed with CT. In our series, myelography disclosed conjoined root anomalies in 2 cases with negative CT scans. With myelography, we have a $100 \%$ rate of diagnosis for this entity. In 9 cases, operative findings supported our preoperative radiological findings.

In 3 Type III and combined anomalies, the precise type was only determinated during surgery.

In the light of our experience and relevant literature we conclude that (1) if nerve root anomalies are diagnosed, then wide decompression is required; (2) failure to recognise root anomalies may lead to excessive traction at operation; and (3) patients who have had 'failed disc surgery' have a high incidence of root anomalies and these should be sought.

\section{References}

1 Zagnoni C (1949) Reperto di un tipo non conosciuto di anastomosi nervosa delle radici spinali. Atti Soc Med-chir Padova 27: 48-52.

2 Epstein JA, Carras R, Ferrar J, Hyman RA, Khan A (1981) Conjoined lumbosacral nerve roots. J Neurosurg 55: 585-589.

3 Gebarski SS, McGillicuddy JE (1984) 'Conjoined' nerve roots: a requirement for computed tomographic and myelographic correlation for diagnosis. Neurosurgery 14 66-68.

4 Helms CA, Dorwart RH, Gray M (1982) The CT appearance of conjoined nerve roots and differentiation from a herniated nucleus pulposus. Radiology 144: 803-807.

5 Neidre A, Macnab I (1983) Anomalies of the lumbosacral nerve roots. Spine 8: 294-299.

6 White JG, Strait TA, Binkley JR, Hunter SE (1982) Surgical treatment of 63 cases of conjoined nerve roots. J Neurosurg 56: 114-117.

7 Cannon BW, Hunter SE, Picaza JA (1961) Nerve root anomalies in lumbar disc surgery. J Neurosurg 19: 208-214.

8 Bouchard JM, Copty M, Langelier R (1978) Preoperative diagnosis of conjoined roots anomaly with herniated lumbar disks. Surg Neurol 10: 229-231.

9 Bernini PM, Wiesel SW, Rothman RH (1980) Metrizamide myelography and the identification of anomalous lumbosacral nerve roots. J Bone Joint Surg 62A 7: 1203-1208. 